\title{
First molecular characterization of Sarcocystis tenella in Tatra chamois (Rupicapra rupicapra tatrica) in Poland
}

\author{
Rafal Kolenda $^{1}$ • Peter Schierack ${ }^{1}$ - Filip Zieba ${ }^{2}$ - Tomasz Zwijacz-Kozica ${ }^{2}$. \\ Michal Bednarski ${ }^{3}$
}

Received: 11 May 2015 / Accepted: 2 July 2015 / Published online: 24 July 2015

(C) The Author(s) 2015. This article is published with open access at Springerlink.com

\begin{abstract}
In this study, sarcocysts from three Polish Tatra chamois were isolated and identified using morphological and molecular methods for the first time. Six cysts were found in the latissimus dorsi muscle and another two in the diaphragm. No sarcocysts were detected in the myocardium, tongue, and esophagus. The isolated cysts were long with rounded ends, $0.35-0.61 \mathrm{~mm}$ in length, and $0.02-0.06 \mathrm{~mm}$ in width. All the sarcocysts were identified as Sarcocystis tenella on the basis of light microscopy and sequencing of cytochrome $\mathrm{C}$ oxidase subunit I ( $\operatorname{cox} I)$ and small-subunit rRNA ( $s s u$ rRNA) genes. Comparative analysis showed a $99.23 \%$ identity of the coxl gene sequences from Tatra chamois and sheep sarcocysts, and an even higher degree of sequence identity $(99.88 \%$ ) was documented in the case of the ssu rRNA gene. When compared at a haplotype level, all the sheep sequences of cox 1 differed from those isolated from Tatra chamois. In contrast, one out of the two ssu rRNA haplotypes from the sheep isolates was identical with the
\end{abstract}

Electronic supplementary material The online version of this article (doi:10.1007/s00436-015-4619-4) contains supplementary material, which is available to authorized users.

Rafał Kolenda

rkolenda@B-TU.de

Michał Bednarski

michal.bednarski@up.wroc.pl

1 Faculty of Natural Sciences, Brandenburg University of Technology Cottbus-Senftenberg, Großenhainer Str. 57,

D-01968 Senftenberg, Germany

2 Tatra National Park, Kuźnice 1, 34-500 Zakopane, Poland

3 Department of Epizootiology and Clinic of Bird and Exotic Animals, Wrocław University of Environmental and Life Sciences, 50-375 Wrocław, Poland haplotype from Tatra chamois. In conclusion, we showed that coxl and ssu rRNA genes can be used as genetic markers for identification of the $S$. tenella, with cox 1 gene providing better resolution during phylogenetic analyses. However, both genetic population analysis and phylogenetic inference with coxl and ssu rRNA genes demonstrated that they do not constitute good markers for spatial differentiation of $S$. tenella.

Keywords Sarcocystis tenella $\cdot$ Tatra chamois $\cdot$ cox $1 \cdot$ ssu $r R N A \cdot$ Phylogenetic analysis

\section{Introduction}

Sarcocystis spp. are protozoan intracellular parasites with a two-host life cycle (Fayer 2004). Their intermediate hosts are herbivores and omnivores, while carnivores constitute the definitive hosts. After ingestion of oocysts by the intermediate host, Sarcocystis spp. undergo asexual generations in the vascular endothelial cells in all parts of the body with eventual formation of mature sarcocysts in the hosts' muscles. The definitive host is infected due to ingestion of the Sarcocystisinfected muscular tissue, which results in the intestinal generation of oocysts (Fayer et al. 2015).

The Bovidae family consists of 143 species (Heller et al. 2013), including many well-established intermediate hosts for Sarcocystis spp. (Tenter 1995). While Sarcocystis spp. found in domesticated Bovidae, e.g., cattle, sheep, and goats, have been characterized extensively (Dubey et al. 1989, 2014; Morsy et al. 2011; Formisano et al. 2013), recent research centers around wild ruminants as intermediate hosts for these protozoa (Dahlgren and Gjerde 2007; Gjerde 2013). However, still little is known about Sarcocystis spp. infection in many wild ruminant species, e.g., in Tatra chamois (Rupicapra rupicapra tatrica). Tatra chamois is listed as a critically 
endangered species in the IUCN Red List of Threatened Species (Aulagnier et al. 2008). The animals from this subspecies can be found in Polish and Slovak Tatra mountains. The population of Tatra chamois was estimated at only 220 in 1999 (Jurdíková 2000) and at 950 in 2012 (Tatra National Park Information). Due to such small size of Tatra chamois population, an information about the ecology and pathology of Sarcocystis spp. in this species becomes of vital importance. While Sarcocystis spp. were demonstrated as a cause of enzootic muscle tissue parasitoses in other ruminants, with significant losses in livestock due to abortions, reduced weight, neurological disease, and even mortality in rarely observed acute cases (Caldow et al. 2000; Schock et al. 2012; Agerholm and Dubey 2014), we still lack a data on the prevalence and pathogenic role of Sarcocystis spp. in Tatra chamois.

Sarcocystis spp. can be identified with morphological and molecular methods. Molecular techniques (Tenter et al. 1994; Morgan and Thompson 1999) have successfully replaced morphological methods as the latter was shown to be more prone to misjudgment. Cytochrome $\mathrm{C}$ oxidase subunit I (coxl) and small-subunit rRNA (ssu rRNA) gene sequencing was the primary molecular technique used in most recently published studies dealing with the problem in question (Dahlgren and Gjerde 2010; Gjerde 2012). The results of these studies can be used for characterization of inter- and intraspecies phylogenetic relationships. However, the question which of these two genes is more suitable for species determination and phylogenetic analyses is still a subject of ongoing discussion (Gjerde 2013). It is noteworthy that the ssu rRNA sequences turned out to be indistinguishable for some species (e.g., Sarcocystis tarandi and Sarcocystis elongata, Sarcocystis rangiferi and Sarcocystis truncata). In contrast, the analysis based on coxl gene sequences provided satisfactory resolution both on the species level and within the phylogenetic inference (Gjerde 2014a).

To the best of our knowledge, no data on the prevalence of Sarcocystis spp. in Tatra chamois have been published to date. Therefore, the aim of this study was to identify Sarcocystis spp. present in Tatra chamois and to compare their sequences for coxl and ssu rRNA genes with those found in other Bovidae. Moreover, our research centered around the phylogenetic placement of the isolated sarcocysts and potential use of coxl and ssu rRNA genes as molecular markers for phylogeographic studies of newly discovered Sarcocystis spp.

\section{Material and methods}

\section{Isolation and morphological identification of sarcocysts}

The study included eight sarcocysts isolated from three adult Tatra chamois individuals found dead in the Polish Tatra National Park during the winter season of 2012 and 2013. The animals did not show any signs of infection and probably died due to multiple injuries caused by avalanches as they presented with broken legs, ribs, and spine. The specimens from the diaphragm and latissimus dorsi muscle (MLD, 200 g), myocardium $(30-40 \mathrm{~g})$, tongue $(10 \mathrm{~g})$, and esophagus $(20-25 \mathrm{~g})$ were examined for the presence of sarcocysts. Individual sarcocysts were excised under a stereomicroscope with an aid of fine needles. A new needle was used for each specimen. Excised sarcocysts were washed three times with PBS and examined under a light microscope (LM). Subsequently, the material was stored at $-20^{\circ} \mathrm{C}$ for up to 6 months until DNA isolation.

\section{DNA isolation, amplification of cox 1 and ssu rRNA genes, cloning, and sequencing}

Total DNA was extracted with a DNeasy Blood \& Tissue Kit (QIAGEN GmbH, Germany). The isolation procedure was performed according to the manufacturer's protocol. The coxl and ssu rRNA genes were amplified by PCR as described previously, with minor modifications (Kolenda et al. 2014). Briefly, the following primers were used for the amplification of coxl and ssu rRNA genes: 5'-CGGTATGTACATACTTAC GGCG-3' and 5'-AAACAAATGCAATGGCTGCC-3' ( $\operatorname{cox} 1)$; 5'-GCCATGCATGTCTAAGTATAAGC-3' and 5'CCTTGTTACGACTTCTCCTTCC-3' (ssu rRNA). A total of $50 \mu \mathrm{l}$ of the reaction mix for each primer pair contained DNA $(2 \mu \mathrm{l})$, Phusion HF buffer (Thermo Scientific), $\mathrm{MgCl}_{2}$ (at final concentration of $4 \mathrm{mM}$ ), $1 \mathrm{U}$ of Phusion High-Fidelity DNA Polymerase (Thermo Scientific), $0.2 \mu \mathrm{M}$ of each primer, and $0.2 \mathrm{mM}$ dNTPs mix. PCR was started with initial denaturation $\left(98^{\circ} \mathrm{C}, 30 \mathrm{~s}\right)$, followed by 40 cycles of denaturation $\left(98^{\circ} \mathrm{C}, 5 \mathrm{~s}\right)$, annealing $\left(56^{\circ} \mathrm{C}, 15 \mathrm{~s}\right)$, elongation $\left(72^{\circ} \mathrm{C}, 1 \mathrm{~kb} /\right.$ $\mathrm{s})$, and final elongation $\left(72{ }^{\circ} \mathrm{C}, 5 \mathrm{~min}\right)$. The PCR products were run on a $1 \%$ agarose gel. Subsequently, the gel was stained with ethidium bromide, and appropriate bands were excised and purified with a MinElute Gel Extraction Kit (QIAGEN, Germany) according to the manufacturer's protocol. The purified DNA was cloned into the vector pJET 1.2 of the CloneJET PCR Cloning Kit (Thermo Scientific) in line with the manufacturer's protocol. One coxl- and one ssu $r R N A$-positive plasmid from each sarcocyst isolate was sent for sequencing.

\section{Sequencing, sequence alignment, comparison, and phylogenetic analyses}

The coxl- and ssu rRNA-positive plasmids were sequenced with the following primers: 5'-CGACTCACTATAGGGAGA GCGGC-3' and 5'-AAGAACATCGATTTTCCATGGCAG$3^{\prime}$ (primer binding sites on the plasmid, used for coxl and ssu rRNA); 5'-TATCCCCATCACGATGCATAC-3' (primer binding site on the ssu rRNA sequences) (Dahlgren and Gjerde 2007). The sequences were determined by a BigDye ${ }^{\circledR}$ 
Terminator v3.1 cycle sequencing (Life Technologies) and analyzed on an ABI fluorescence automated DNA sequencer at the LGC Genomics (Berlin, Germany). The sequence reads were assembled into contigs with a CAP3 software (Huang and Madan 1999).

The coxl and ssu rRNA gene sequences were aligned using the default settings of the ClustalX 2.1 software (Larkin et al. 2007). The alignments for coxl and ssu rRNA genes, consisting of 375 and 184 sequences, respectively (Table S1), were used for further phylogenetic analyses.

The coxl and ssu rRNA gene sequences for Sarcocystis tenella were compared using an Arlequin 3.5 software (Excoffier and Lischer 2010) and MEGA 6.06 software (Tamura et al. 2013). We analyzed the number of haplotypes $(\mathrm{H})$, number of observed substitutions (S), transitions (Trs) and transversions (Trv), nucleotide diversity $(\pi)$, average number of pairwise differences (Theta- $\pi$ ), and mean pairwise distance between the groups/species. The inter-haplotypic distance matrices were generated with an R package (R Development Core Team 2013).

The phylogenetic reconstruction based on a maximum likelihood (ML) and maximum parsimony (MP) was conducted with a MEGA 6.06 software (Tamura et al. 2013). GTR $+\mathrm{G}+\mathrm{I}$ and $\mathrm{K} 2+\mathrm{G}+\mathrm{I}$ were identified as the optimal substitution models for the coxl and ssu rRNA ML phylograms, respectively. In the case of the ssu rRNA gene, the analyses did not include gaps in alignment. The MP trees for both genes were created with using a subtree-pruning-regrafting (SPR) algorithm. The reliability of the ML and MP phylograms was verified with the bootstrap method. MrBayes v. 3.2 was used for the Bayesian tree construction (Ronquist et al. 2012). The Bayesian searches for the coxl gene were initiated with random starting trees based on a GTR $+\mathrm{G}+\mathrm{I}$ model for 14,000 , 000 generations, and the tree was sampled every 500 generations. The Bayesian searches for the ssu rRNA gene were initiated with random starting trees based on a $\mathrm{K} 2+\mathrm{G}+\mathrm{I}$ model for 10,000,000 generations, and the tree was sampled every 500 generations. The burn-in value for coxl and ssu rRNA genes was set at $25 \%$.

\section{Results}

\section{Isolation and morphological characterization of sarcocysts}

The samples from three adult Tatra chamois were examined for the presence of sarcocysts. Six cysts were found in the latissimus dorsi muscle and another two were isolated from the diaphragm specimens (Table 1). We did not find any sarcocysts in the myocardium, tongue, and esophagus. No macroscopic cysts were found. All the isolated cysts were long with rounded ends, $0.35-0.61 \mathrm{~mm}$ in length and 0.02 $0.06 \mathrm{~mm}$ in width. The sarcocysts were not encapsulated by a fibrous material. On light microscopy, the cysts appeared to have smooth (lacking a fibrillary structure) thin walls without any protrusions (Fig. S1).

\section{Molecular characterization of the coxl gene}

We obtained eight sequences and compared them with the sequences stored in the GenBank. All the sequences were eventually identified as $S$. tenella and submitted to the GenBank under the accession numbers KP263744KP263751.

Subsequently, we compared our cox 1 sequences from Tatra chamois with the available sequences of $S$. tenella cox 1 gene (GenBank Accession No. KC209723-KC209732). All the sequences stored in the GenBank originated from sheep sarcocysts. We found 12 polymorphic sites in the eight coxl sequences from Tatra chamois and 33 polymorphic sites in the ten available coxl sequences from sheep (Table S2). Compared to the sheep sequences, the sequences from Tatra chamois showed nearly twofold lower nucleotide diversity and average number of pairwise differences per sequence (Table 2). Moreover, the number of private substitutions in the sheep sarcocysts was 3.5 -folds higher than in the sarcocysts from Tatra chamois. When the sequences were compared at a haplotype level, all the sheep haplotypes of Sarcocystis differed from the Tatra chamois haplotypes (Fig. S2). While all the
Table 1 Morphological characterization of Sarcocystis tenella isolated from Tatra chamois and the GenBank accession numbers for coxl and ssu rRNA genes

\begin{tabular}{llllll}
\hline Animal number/sex & Time of isolation & Muscle & Size [mm] & \multicolumn{2}{l}{ GenBank accession nos. } \\
\cline { 5 - 6 } & & & & cox1 & ssu rRNA \\
\hline 1/female & March 2012 & MLD & $0.35 \times 0.02$ & KP263746 & KP263754 \\
& & & $0.42 \times 0.03$ & KP263747 & KP263755 \\
& & Diaphragm & $0.58 \times 0.05$ & KP263748 & KP263756 \\
2/male & October 2012 & MLD & $0.61 \times 0.05$ & KP263749 & KP263757 \\
& & Diaphragm & $0.46 \times 0.04$ & KP263750 & KP263758 \\
& & MLD & $0.39 \times 0.03$ & KP263744 & KP263752 \\
& & & $0.44 \times 0.03$ & KP263745 & KP263753 \\
3/male & February 2013 & MLD & $0.53 \times 0.06$ & KP263751 & KP263759 \\
\hline
\end{tabular}


Table 2 Molecular diversity of coxl and ssu rRNA genes from S. tenella

\begin{tabular}{|c|c|c|c|c|c|c|}
\hline & \multicolumn{2}{|l|}{ Tatra chamois } & \multicolumn{2}{|l|}{ Sheep } & \multicolumn{2}{|l|}{ Together } \\
\hline & $\operatorname{cox} 1$ & ssu rRNA & $\operatorname{cox} 1$ & ssu rRNA & $\operatorname{cox} 1$ & ssu rRNA \\
\hline $\mathrm{N}$ & 8 & 8 & 10 & 4 & 18 & 12 \\
\hline $\mathrm{H}$ & 6 & 7 & 10 & 2 & 16 & 8 \\
\hline $\mathrm{P}$ & 12 & 10 & 33 & 1 & 41 & 10 \\
\hline $\mathrm{S}$ & 12 & 10 & 33 & 1 & 41 & 10 \\
\hline Trs & 10 & 10 & 21 & 1 & 28 & 10 \\
\hline $\operatorname{Trv}$ & 2 & 0 & 12 & 0 & 13 & 0 \\
\hline $\mathrm{pS}$ & 8 & 9 & 29 & 0 & - & - \\
\hline$\pi( \pm \mathrm{SD})$ & $0.0048( \pm 0.003)$ & $0.002( \pm 0.0013)$ & $0.009( \pm 0.0051)$ & $0.0003( \pm 0.0003)$ & $0.0075( \pm 0.0041)$ & $0.0014( \pm 0.0009)$ \\
\hline Theta- $\pi( \pm \mathrm{SD})$ & $4.75( \pm 2.60)$ & $3.54( \pm 2.01)$ & $8.87( \pm 4.47)$ & $0.50( \pm 0.52)$ & $7.44( \pm 3.65)$ & $2.61( \pm 1.50)$ \\
\hline
\end{tabular}

$N$ number of isolates, $H$ number of haplotypes, $P$ number of polymorphic sites, $S$ number of substitution sites, Trs number of transitions, Trv number of transversions, $p S$ private substitutions sites, $\pi$ nucleotide diversity, Theta- $\pi$ average number of pairwise differences

sheep haplotypes were different, four sequences from Tatra chamois belonged to two haplotypes.

The phylogenetic analyses of the coxl gene sequences from Sarcocystis spp. isolated from cattle, cervids, and sheep were conducted with three methods: ML, MP, and Bayesian inference. As the trees with highly similar clade arrangements were obtained irrespective of the method used, we present only the ML tree (Fig. 1). All the Polish coxl sequences from $S$. tenella are placed alongside the sequences from sheep in one cluster. All the sequences from Tatra chamois can be found in separate nodes of this cluster, but they do not tend to group in a single subclade. These findings are further supported by low pairwise distance values between the groups, as shown in Table 3. The Norwegian isolates showed higher intragroup pairwise distance $(0.009 \pm 0.002)$ as compared to the intergroup pairwise distance between the Polish and Norwegian isolates $(0.008 \pm 0.002)$.

\section{Molecular characterization of the ssu $r R N A$ gene}

We obtained eight sequences and compared them with the sequences stored in the GenBank. All the sequences were eventually identified as $S$. tenella and submitted to the GenBank under the accession numbers shown in Table 1.

Similar as in the case of the coxl gene, the sSu rRNA sequences from Polish Tatra chamois were compared with the available $S$. tenella ssu rRNA gene sequences (GenBank Accession No. KC209734-KC209737). All the GenBank sequences were obtained from sheep sarcocysts from Norway. The ssu rRNA sequences isolated from Polish specimens contained ten polymorphic sites as compared to only one polymorphic site in the Norwegian isolates (Table S3). Three sheep sequences (KC209734-KC209736) and one sequence from Tatra chamois (KP263759) turned out to be identical. The average number of pairwise differences and nucleotide diversity in the sequences from Tatra chamois were approximately sevenfolds higher compared to the sheep sequences (Table 2). The analysis at a haplotype level revealed that one out of the two haplotypes from sheep isolates was identical with the haplotype from Tatra chamois (Fig. S3).

The phylogenetic relationships between the ssu rRNA genes of Sarcocystis spp. specimens isolated from cervids, cattle, and sheep were analyzed on the basis of ML, MP, and Bayesian inference. While the trees obtained with the three methods generally showed similar topologies, two principal differences were documented. Firstly, we found high intermethod variance in branching for the clade consisting of $S$. tarandi, S. truncata, S. sp., S. rangiferi, S. elongata, and Sarcocystis silva, and none of the methods was able to group the sequences of the same species together. As the problem was reported previously (Gjerde 2013) and did not directly influence our findings, we eventually did not include this part of the tree in further analyses. Secondly, the three methods used for the phylogenetic reconstruction produced different results regarding the relationship between $S$. tenella and Sarcocystis capracanis. While only one $S$. tenella ssu rRNA sequence (KP263755) clustered together in with the sequence from $S$. capracanis (L76472) in the MP and ML trees, the latter sequence was clustered with all the $S$. tenella sequences in the tree constructed with the Bayesian inference.

\section{Discussion}

Sarcocystis spp. are common parasites of cervids, cattle, and sheep. S. tenella was characterized well as a parasite of sheep and is an established etiological factor of enzootic muscle tissue parasitoses and neurological disorders, mainly in lambs. In this study, we used light microscopy, coxl and ssu rRNA gene sequencing, genetic population analysis, and 


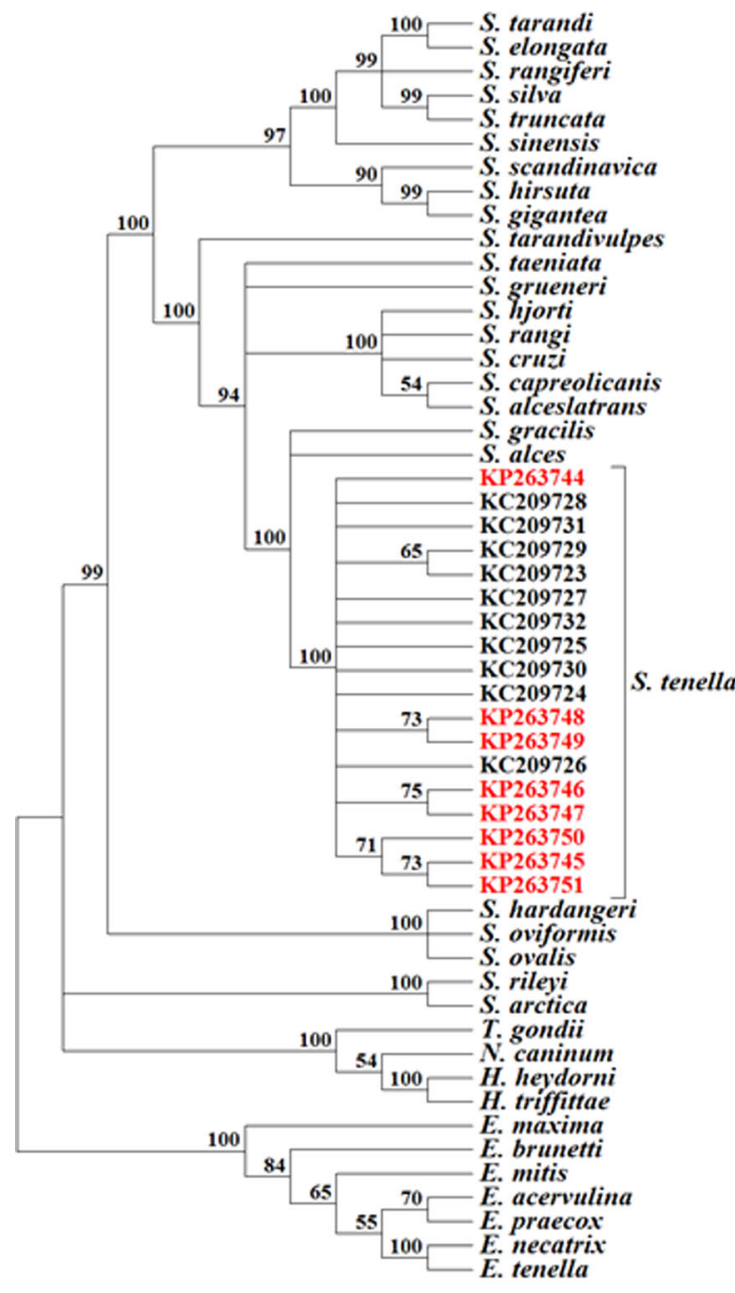

a

Fig. 1 Maximum likelihood for DNA phylograms of selected Sarcocystidae and Coccidia. a The coxl tree was constructed based on an alignment of partial coxl gene sequences from eight Polish Sarcocystis tenella isolates and available coxl gene sequences of related species deposited in the GenBank (a total of 375 sequences). b The ssu rRNA tree was constructed based on an alignment of nearly complete ssu rRNA gene sequences from eight Polish $S$. tenella isolates and available ssu

phylogenetic inference to identify and characterize $S$. tenella isolated from Polish Tatra chamois. To the best of our knowledge, this was the first study to identify Sarcocystis spp. from Tatra chamois at a molecular level.

Although we examined various tissues of three adult Tatra chamois, all the eight sarcocysts were isolated solely from the diaphragm and latissimus dorsi muscle specimens. In contrast, the authors of previous studies involving sheep (Pomroy and Charleston 1987; Dubey et al. 1988; Oryan et al. 1996) isolated $S$. tenella also from all other tissues that have been examined during the course of our experiment. Morphology of all the isolated cysts matched with the previously described characteristics of $S$. tenella cysts from sheep, obtained up to 60 days postinfection with this parasite (Mehlhorn et al.

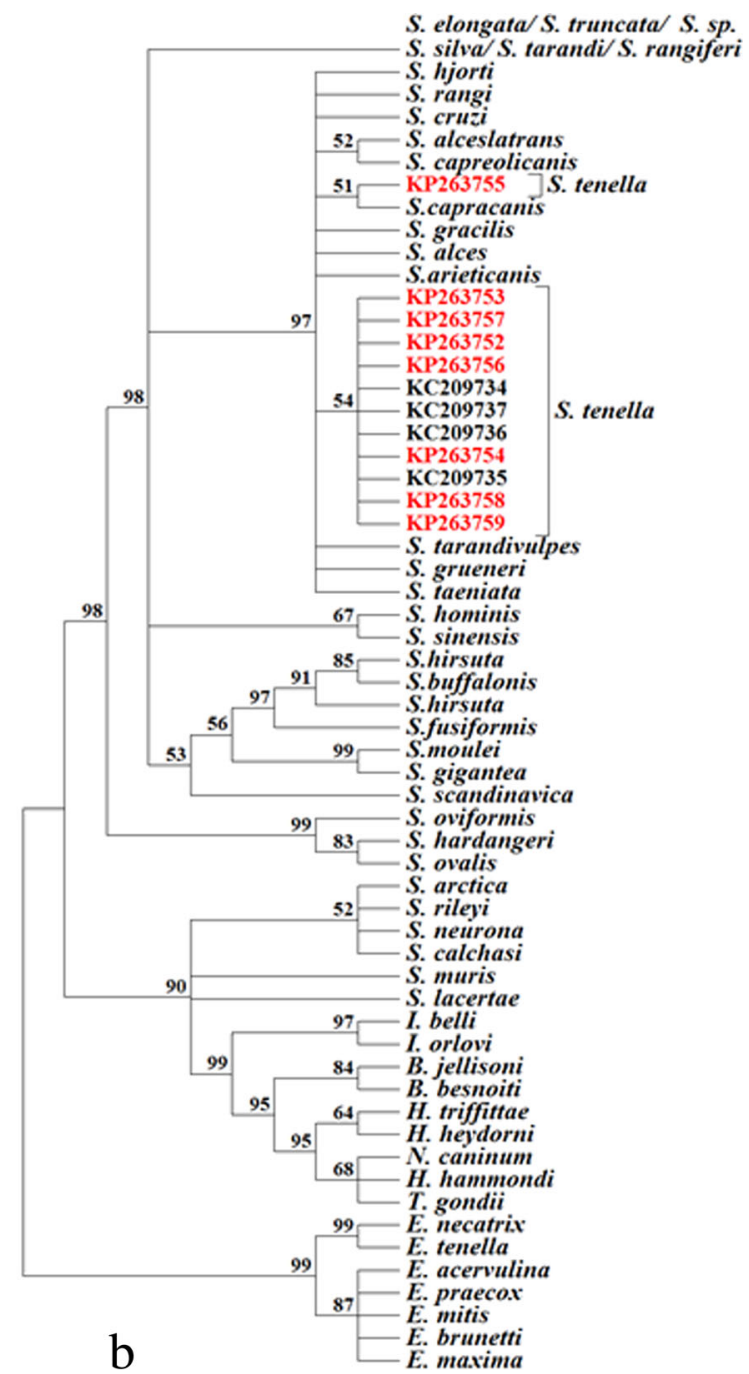

$r R N A$ gene sequences of related species deposited in the GenBank (a total 184 sequences). The trees were rooted with Eimeria spp. The values between the branches represent percent bootstrap value per 1000 replicates. Bootstrap values below $50 \%$ are not shown. Polish isolates are marked in red. The GenBank accession numbers of all sequences used for construction of the trees are given in Table S1

1975). Older $S$. tenella cysts isolated from sheep were shown to present with fine palisade-like protrusions (Mehlhorn et al. 1975), not observed in the case of our sarcocysts from Tatra chamois. To the best of our knowledge, only two cases of Sarcocystis spp. invasion in Alpine chamois have been reported thus far (Boch and Schneidawind 1988; Odening et al. 1996). Boch and Schneidawind described three Sarcocystis types in Alpine chamois, including one identified morphologically as S. tenella. In turn, Odening et al. (1996) isolated two morphological types of Sarcocystis, which they referred to as $S$. sp. and Sarcocystis cornagliai.

Although $S$. tenella was demonstrated to cause a severe disease in sheep (Munday et al. 1975; Caldow et al. 2000; Schock et al. 2012; Agerholm and Dubey 2014), we cannot 


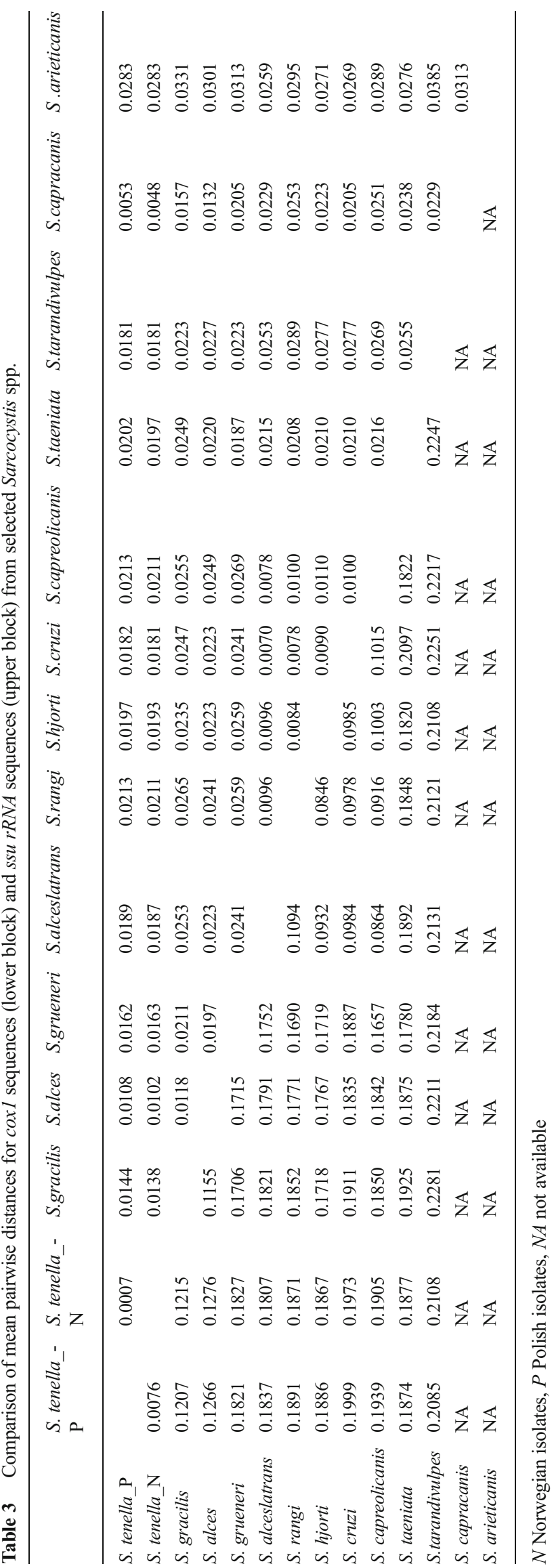

speculate on a potential pathogenicity of this species in Tatra chamois due to a small number of examined cadaveric specimens and the fact that we found only few microscopic cysts in the analyzed tissues.

Based on the analysis of coxl and ssu rRNA gene sequences from the eight sarcocysts isolated from Polish Tatra chamois, we identified them as the cysts of $S$. tenella. To this date, this parasite was isolated exclusively from sheep. The comparative analysis of our coxl gene sequences from Tatra chamois and the sequences from sheep sarcocysts described by Gjerde (2013) revealed that the latter presented with a higher number of both overall and private substitutions. In contrast, our ssu rRNA sequences from Tatra chamois contained more substitutions than the sheep sequences. Such high variability of the ssu rRNA sequences from Polish $S$. tenella is rather surprising as our previous study of other Sarcocystis spp. (Kolenda et al. 2014) revealed that this gene is highly conservative among the isolates from a single population. Previous research on Eimeria and Sarcocystis spp. (Ogedengbe et al. 2011; Gjerde 2014a; Kolenda et al. 2014) showed that coxl gene provides higher resolution of interspecies genetic differences than ssu rRNA. We obtained similar results during the comparative analysis of $S$. tenella and other closely related taxa (Table 3 ).

One aim of our study was to verify if coxl and/or ssu rRNA genes can be used as molecular markers for phylogeographic studies of S. tenella. Therefore, we compared the sequences of these genes from Polish and Norwegian specimens at a haplotype level (Figs. S2 and S3) and subjected them to a phylogenetic reconstruction (Fig. 1). All the Polish coxl sequences could be separated from their Norwegian counterparts at a haplotype level. Unfortunately, the phylogenetic analysis revealed that the differences between the Polish and Norwegian sequences were too subtle to discriminate their geographic origin from an evolutionary tree. Furthermore, the two groups of sequences could not be distinguished on the basis of mean pairwise distance matrix (Table 3). Also, the results for ssu $r R N A$ sequences were not satisfactory. One haplotype from Tatra chamois turned out to be identical with the sheep haplotype, and we were unable to distinguish between the sequences with different geographic origin on the basis of their phylogenetic reconstruction. Moreover, the evolutionary trees obtained with various methods branched $S$. capracanis with $S$. tenella isolates in a different manner. Poor performance of evolutionary trees for the ssu rRNA gene sequences was previously reported by Gjerde. According to this author, this likely resulted from the difficulties in aligning hypervariable regions of ssu rRNA sequences between different Sarcocystistidae species (Gjerde 2014a). The analysis of mean pairwise distance matrix for the ssu rRNA sequences confirmed the results obtained during genetic population and phylogenetic analyses (Table 3). Due to lack of sequences from S. tenella isolated from sheep living in the area inhabited by 
Tatra chamois and poor performance of coxl and/or ssu rRNA genes as molecular markers for phylogeographic studies, we were unable to conclude if the minor sequence differences between Tatra chamois and Norwegian sheep isolates reflected the presence of country-specific variants or rather differences between the hosts.

Our findings provide a novel insight into intermediate host range for $S$. tenella. While $S$. tenella was only reported in sheep thus far (Mehlhorn et al. 1975; Haziroglu et al. 2002; Rassouli et al. 2014), we showed that also wildlife animals may be a reservoir of this parasite. Both sheep and chamois are members of the Caprinae subfamily, which implies that also other species from this group are susceptible to $S$. tenella infection. Our findings are consistent with the results of several recent studies of game animals, which have also found that the same Sarcocystis sp. might occur in more than one host (Dahlgren and Gjerde 2007, 2010; Gjerde 2014b). Previous studies showed that final hosts for $S$. tenella are canids (Dubey et al. 1982), and probably dogs and foxes are final hosts for this parasite in the region of Tatra mountains. Still, more work should be done to confirm life cycle stages and transmission of this pathogen between intermediate hosts and final hosts in Tatra mountains.

In summary, this is the first study to demonstrate that Tatra chamois can be an intermediate host for S. tenella. Our findings suggest that coxl and ssu rRNA genes can be used as genetic markers for identification of $S$. tenella, with coxl gene providing better resolution during phylogenetic analyses. Furthermore, we showed that coxl and ssu rRNA genes do not perform well as molecular markers for phylogeographic studies of S. tenella.

Acknowledgments This study was supported by the Federal Ministry of Education and Research, Germany [Project InnoProfile-Transfer 03IP611X].

Open Access This article is distributed under the terms of the Creative Commons Attribution 4.0 International License (http:// creativecommons.org/licenses/by/4.0/), which permits unrestricted use, distribution, and reproduction in any medium, provided you give appropriate credit to the original author(s) and the source, provide a link to the Creative Commons license, and indicate if changes were made.

\section{References}

Agerholm JS, Dubey JP (2014) Sarcocystosis in a stillborn lamb. Reprod Domest Anim 49:e60-e63. doi:10.1111/rda.12398

Aulagnier S, Giannatos G, Herrero J (2008) Rupicapra rupicapra. The IUCN Red List of Threatened Species. Version 2014.3. www. iucnredlist.org

Boch J, Schneidawind H (1988) Krankheiten des jagdbaren Wildes. Parey, Hamburg
Caldow GL, Gidlow JR, Schock A (2000) Clinical, pathological and epidemiological findings in three outbreaks of ovine protozoan myeloencephalitis. Vet Rec 146:7-10

Dahlgren SS, Gjerde B (2007) Genetic characterisation of six Sarcocystis species from reindeer (Rangifer tarandus tarandus) in Norway based on the small subunit rRNA gene. Vet Parasitol 146:204213. doi:10.1016/j.vetpar.2007.02.023

Dahlgren SS, Gjerde B (2010) Molecular characterization of five Sarcocystis species in red deer (Cervus elaphus), including Sarcocystis hjorti n. sp., reveals that these species are not intermediate host specific. Parasitology 137:815-840. doi:10.1017/ S0031182009991569

Dubey JP, Speer CA, Callis G, Blixt JA (1982) Development of the sheep-canid cycle of Sarcocystis tenella. Can J Zool 60:2464 2477. doi:10.1139/z82-315

Dubey JP, Lindsay DS, Speer CA et al (1988) Sarcocystis arieticanis and other Sarcocystis species in sheep in the United States. J Parasitol 74:1033-1038

Dubey JP, Speer CA, Fayer R (1989) Sarcocystosis of animals and man. $\mathrm{X}+215 \mathrm{pp}$

Dubey JP, Fayer R, Rosenthal BM et al (2014) Identity of Sarcocystis species of the water buffalo (Bubalus bubalis) and cattle (Bos taurus) and the suppression of Sarcocystis sinensis as a nomen nudum. Vet Parasitol 205:1-6. doi:10.1016/j.vetpar.2014.06.020

Excoffier L, Lischer HEL (2010) Arlequin suite ver 3.5: a new series of programs to perform population genetics analyses under Linux and Windows. Mol Ecol Resour 10:564-567. doi:10.1111/j.1755-0998. 2010.02847.x

Fayer R (2004) Sarcocystis spp. in human infections. Clin Microbiol Rev 17:894-902. doi:10.1128/CMR.17.4.894-902.2004

Fayer R, Esposito DH, Dubey JP (2015) Human infections with Sarcocystis species. Clin Microbiol Rev 28:295-311. doi:10.1128/ CMR.00113-14

Formisano P, Aldridge B, Alony Y et al (2013) Identification of Sarcocystis capracanis in cerebrospinal fluid from sheep with neurological disease. Vet Parasitol 193:252-255. doi:10.1016/j.vetpar. 2012.12.016

Gjerde B (2012) Morphological and molecular characterization and phylogenetic placement of Sarcocystis capreolicanis and Sarcocystis silva n. sp. from roe deer (Capreolus capreolus) in Norway. Parasitol Res 110:1225-1237. doi:10.1007/s00436-011-2619-6

Gjerde B (2013) Phylogenetic relationships among Sarcocystis species in cervids, cattle and sheep inferred from the mitochondrial cytochrome c oxidase subunit I gene. Int J Parasitol 43:579-591. doi: 10.1016/j.ijpara.2013.02.004

Gjerde B (2014a) Sarcocystis species in red deer revisited: with a redescription of two known species as Sarcocystis elongata n. sp. and Sarcocystis truncata n. sp. based on mitochondrial coxl sequences. Parasitology 141:441-452. doi:10.1017/ S0031182013001819

Gjerde B (2014b) Morphological and molecular characteristics of four Sarcocystis spp. in Canadian moose (Alces alces), including Sarcocystis taeniata n. sp. Parasitol Res 113:1591-1604. doi:10. 1007/s00436-014-3806-Z

Haziroglu R, Guvenc T, Tunca R (2002) Electron microscopical studies on cysts of Sarcocystis arieticanis within cardiac muscle of naturally infected sheep. Parasitol Res 89:23-25. doi:10.1007/s00436-0010549-4

Heller R, Frandsen P, Lorenzen ED, Siegismund HR (2013) Are there really twice as many bovid species as we thought? Syst Biol 62: 490-493. doi:10.1093/sysbio/syt004

Huang X, Madan A (1999) CAP3: a DNA sequence assembly program. Genome Res 9:868-877

Jurdíková N (2000) The decline of the Tatra chamois. Caprinae, Newsletter of the IUCN/SSC Caprinae Specialist Group 
Kolenda R, Ugorski M, Bednarski M (2014) Molecular characterization of Sarcocystis species from Polish roe deer based on ssu rRNA and cox1 sequence analysis. Parasitol Res 113:3029-3039. doi:10.1007/ s00436-014-3966-X

Larkin MA, Blackshields G, Brown NP et al (2007) Clustal W and Clustal $X$ version 2.0. Bioinformatics 23:2947-2948. doi:10.1093/ bioinformatics/btm 404

Mehlhorn H, Heydorn AO, Gestrich R (1975) Light and electron microscope studies on cysts of Sarcocystis ovicanis Heydorn et al. (1975) within muscle fibres of sheep (author's transl). Parasitol Res 48:8393

Morgan UM, Thompson RCA (1999) Molecular detection of parasitic protozoa. Parasitology 117:73-85

Morsy K, Saleh A, Al-Ghamdi A et al (2011) Prevalence pattern and biology of Sarcocystis capracanis infection in the Egyptian goats: a light and ultrastructural study. Vet Parasitol 181:75-82. doi:10. 1016/j.vetpar.2011.05.010

Munday BL, Barker IK, Rickard MD (1975) The developmental cycle of a species of Sarcocystis occurring in dogs and sheep, with observations on pathogenicity in the intermediate host. Parasitol Res 46: $111-123$

Odening K, Stolte M, Bockhardt I (1996) On the diagnostics of Sarcocystis in chamois Rupicapra rupicapra. Appl Parasitol 153160

Ogedengbe JD, Hanner RH, Barta JR (2011) DNA barcoding identifies Eimeria species and contributes to the phylogenetics of coccidian parasites (Eimeriorina, Apicomplexa, Alveolata). Int J Parasitol 41: 843-850. doi:10.1016/j.ijpara.2011.03.007
Oryan A, Moghaddar N, Gaur SN (1996) The distribution pattern of Sarcocystis species, their transmission and pathogenesis in sheep in Fars Province of Iran. Vet Res Commun 20:243-253

Pomroy WE, Charleston WA (1987) Prevalence of dog-derived Sarcocystis spp. in some New Zealand lambs. N Z Vet J 35:141142. doi:10.1080/00480169.1987.35417

Rassouli M, Ahmadpanahi J, Alvandi A (2014) Prevalence of Sarcocystis spp. and Hammondia spp. microcysts in esophagus tissue of sheep and cattle, emphasized on their morphological differences. Parasitol Res 113:3801-3805. doi:10.1007/s00436-014-4047-x

R Development Core Team R Development Core Team (2013). R: a language and environment for statistical computing. R Foundation for Statistical Computing, Vienna, Austria. ISBN 3-900051-07-0, URL http://www.R-project.org

Ronquist F, Teslenko M, van der Mark P et al (2012) MrBayes 3.2: efficient Bayesian phylogenetic inference and model choice across a large model space. Syst Biol 61:539-542. doi:10.1093/sysbio/sys029

Schock A, French H, Chianini F et al (2012) Respiratory disease due to acute Sarcocystis tenella infection in sheep. Vet Rec 170:571. doi: 10.1136/vr.e3796

Tamura K, Stecher G, Peterson D et al (2013) MEGA6: Molecular Evolutionary Genetics Analysis version 6.0. Mol Biol Evol 30: 2725-2729. doi:10.1093/molbev/mst197

Tenter AM (1995) Current research on Sarcocystis species of domestic animals. Int J Parasitol 25:1311-1330

Tenter AM, Luton K, Johnson AM (1994) Species-specific identification of Sarcocystis and Toxoplasma by PCR amplification of small subunit ribosomal RNA gene fragments. Appl Parasitol 35:173-188 